\title{
Repeated infections with Schistosoma mansoni and liver fibrosis in undernourished mice
}

\author{
Eridan M. Coutinho ${ }^{a}{ }^{*}$, Fabiana L. Silva ${ }^{a}$, Andreia F. Barros ${ }^{a}$, Roni E. Araújo ${ }^{a}$, \\ Sheilla A. Oliveira ${ }^{\mathrm{a}}$, Carlos F. Luna ${ }^{\mathrm{a}}$, Aryon A. Barbosa Jr. ${ }^{\mathrm{b}}$, Zilton A. Andrade ${ }^{\mathrm{b}}$ \\ a Laboratory of Immunopathology, Department of Immunology, Aggeu Magalhães Research Center, Oswaldo Cruz Foundation, \\ Av. Moraes Rego s/n, Cidade Universitária, Recife, PE, CEP 50670-420, Brazil \\ ${ }^{\mathrm{b}}$ Laboratory of Experimental Pathology, Gonçalo Moniz Research Center, Oswaldo Cruz Foundation, Rua Waldemar Falcão, \\ 121 Brotas, Salvador, BA, CEP 40295-001, Brazil
}

Received 20 September 2005; received in revised form 16 March 2006; accepted 28 March 2006

Available online 27 December 2006

\begin{abstract}
The mouse model of schistosomal periportal fibrosis (Symmers' "pipestem" fibrosis), that develops in 30-50\% of the infected animals, is not reproduced in undernourished mice. Host nutritional status is likely to be a variable that may influence the outcome and progression of infection, since it interferes with the dynamics of connective tissue changes occurring in chronic hepatic schistosomiasis. Re-infections increase the occurrence of periportal liver fibrosis in well-nourished animals, but it is not known how undernourished mice would behave being repeatedly re-infected. So, 21-day-old male albino Swiss mice were individually exposed to 30 cercariae (percutaneous route) of the BH strain of Schistosoma mansoni, 4 weeks after being on a low-protein diet. Control animals were fed on a commercial balanced chow for mice. The nutritional status was evaluated by body weight gain and measurement of food intake. Mice were divided into four groups: A1 (undernourished, single infected), A2 (well-nourished, single infected), B1 (undernourished, re-infected), B2 (well-nourished, re-infected). The primary infection was performed 4 weeks after ingesting the respective diet. Re-infections started 45 days later, with exposure to 15 cercariae, at 15 day intervals. Mice were sacrificed 18 weeks after the primary exposure. The livers were submitted to morphological (gross and microscopic pathology), morphometric (percentage of fibrosis; granuloma size; volume and numerical densities) by using semi-automatic morphometry, and biochemical (quantification of collagen as hydroxyproline) studies. Worm burdens and hepatic egg counting were also recorded. Values for body weight gains were always lower in undernourished mice, the effects of re-infection being minimal on this regard. Liver and spleen weights were higher in well-nourished mice (either single infected or re-infected) and mainly related to the type of ingested diet. A greater number of re-infected well-nourished mice developed periportal fibrosis, but undernourished re-infected animals did not reproduce this lesion. The percentage of fibrosis and hepatic collagen content were higher in well-nourished mice, but differences between single infected and re-infected groups were not statistically significant.
\end{abstract}

(c) 2006 Elsevier B.V. All rights reserved.

Keywords: Schistosomiasis; Liver fibrosis; Re-infections; Malnutrition

\footnotetext{
* Corresponding author. Tel.: +55 8121012582 ; fax: +558134532449 .

E-mail address: eridan@cpqam.fiocruz.br (E.M. Coutinho).
}

\section{Introduction}

Hepatic periportal "pipestem" fibrosis (Symmers, 1904) is the hallmark of hepatosplenic schistosomiasis. Factors related either to the host (immunological 
response, nutritional status, age, etc.) or to the parasite (strain, intensity of infection, number of re-infections with homologous or heterologous strains) have been investigated.

Henderson et al. (1993) reported that inbred male CBA/J mice chronically infected with Schistosoma mansoni develop two distinct syndromes that imitate the intestinal and the hepatosplenic clinical forms of the human disease, both pathologically and immunologically. However, Silva et al. (2004) reported that the two histological aspects seen in chronic schistosomiasis of the mouse (scattered granulomas, periportal fibrosis) had similar immunological profiles in BALB/c mice.

Well-nourished albino Swiss mice submitted to relatively mild (one to two worm pairs) and prolonged infections with $S$. mansoni (16 weeks or more) develop a lesion that mimics human Symmers' fibrosis (Warren, 1966) as a consequence of a massive and continuous deposition of eggs along the dilated intra-hepatic venous portal branches (Andrade, 1987) in a certain percentage of chronically infected mice (Andrade and Cheever, 1993), However, mice maintained under the same conditions and fed a low-protein diet completely failed to develop such "pipestem"-like portal lesion (Coutinho et al., 1997).

Epidemiological and clinical data taken from human populations in endemic areas suggest that re-infections represent an important risk factor for the development of severe hepatosplenic disease (Coura et al., 1974; Coura, 1975). Successful reproduction of periportal liver fibrosis in the chimpanzee monkey model was only obtained after repeated infections (von Lichtemberg and Sadun, 1968). On the other hand, "pipestem" fibrosis of the liver develops more frequently in mice submitted to repeated infections than in controls with single $S$. mansoni infection (Santos et al., 2000).

Experimental trials on the role of leptin in the pathogenesis of hepatic fibrosis were recently reported by Potter and Mezey (2002) and Potter et al. (2003). This hormone, expressed and secreted by adipocytes and also detected in liver activated stellate cells after culture, proved to be a potentiating but not an essential factor for the development of liver fibrosis produced by $S$. mansoni and carbon tetrachloride administration in mice.

As part of serial studies that are being carried out in our laboratories on the role of nutrition as an additional risk factor in the pathogenesis of schistosomal fibrosis of the liver, this investigation inquires whether "pipestem" fibrosis develops in undernourished mice submitted to repeated $S$. mansoni cercarial exposures.

\section{Materials and methods}

\subsection{Animals}

Male albino Swiss mice (21 days old), weighing $11-15 \mathrm{~g}$, were kept in individual wire bottom cages. They were raised and maintained at the animal facilities of Aggeu Magalhães Research Center (FIOCRUZ). Water and food were provided "ad libitum". Mice were maintained in a temperature- and light-controlled environment $\left(23^{\circ} \mathrm{C}\right)$. All animals received human care in compliance with the guidelines of the Animal Care and Use Committee of the Oswaldo Cruz Foundation (Fiocruz).

\subsection{Infection}

Each mouse was exposed to 30 cercariae (percutaneous route) of the BH strain (Belo Horizonte, Brazil) of $S$. mansoni 4 weeks after the experimental diet intake, according to the respective group. The cercariae were obtained from laboratory raised and infected Biomphalaria glabrata. Infection lasted 18 weeks and the effects of diet intake were followed for 22 weeks.

\subsection{Diets}

Undernutrition was induced by feeding mice with a multideficient and essentially low-protein natural diet (7-8\% protein), planned to simulate that usually ingested by low-income individuals living in endemic areas of Manson's schistosomiasis in Northeast Brazil (Coutinho et al., 1997) and is thus referred to as regional basic diet (RBD). Control diet (NUVILAB) was a pelleted commercial balanced chow for mice (Nuvital Nutrientes Ltd., Colombo, PR, Brazil), with $22 \%$ protein content.

\subsection{Evaluation of nutritional status}

Body weight was weekly recorded and food consumption was measured every day. As this experimental model of mouse undernutrition has been extensively studied in previous investigations (Coutinho, 1980; Teodósio et al., 1990; Coutinho et al., 1992, 1997, 2003), it was now considered unnecessary to evaluate other parameters.

\subsection{Experimental design}

Animals were divided into the following groups, each one containing 10 mice:

- A1: undernourished, single infection;

- A2: well-nourished (normal feeding), single infection; 
- B1: undernourished, re-infected;

- B2: well-nourished (normal feeding), re-infected.

The animals were first infected 4 weeks after ingesting their respective diets. In groups B1 and B2, re-infections started 45 days after the primary infection (5 re-infections with 15 cercariae per mouse, at 15 day intervals). Mice were sacrificed by cervical dislocation thirty days after the last re-infection. All animals, including those with single infections, were sacrificed 18 weeks after the primary exposure.

\subsection{Morphological studies}

The livers were removed, rinsed with PBS (phosphate buffered saline), weighed, and divided into several portions. One sample was placed in Bouin's fixative and/or in buffered ( $\mathrm{pH}$ 7.4) $10 \%$ formaldehyde for histologic examination. Tissue was embedded in paraffin and the $5 \mu \mathrm{m}$ thick sections obtained were stained with hematoxylin-eosin and picrosirius-red method for collagen (Junqueira et al., 1979). A portion of the liver was placed in $4 \%$ potassium hydroxide for egg counting (Cheever, 1968, 1970) and another sample was frozen at $-70{ }^{\circ} \mathrm{C}$ for further biochemical quantification of collagen as hydroxyproline.

Spleens were also removed and weighed for gross studies.

\subsection{Morphometry}

Randomly sampled $5 \mu \mathrm{m}$-thick liver histological sections stained with picrosirius-red for collagen, were examined by semiautomatic morphometry using the LEICA QWIN Plus (2.8) Image Processing and Analysis System (Leica Cambridge, Cambridge, England) attached to a JVC TK 1380 digital camera. For morphometric measurements a total sectional area of $4.9 \mathrm{~mm}^{2}$ per animal was evaluated. All granulomas around schistosome eggs or egg shells were included. A spherical shape and normal size distribution were assumed. The following parameters were calculated for the granulomas: size, volume density $\left(V_{\mathrm{v}}\right)$, and numerical density $\left(N_{\mathrm{n}}\right)$. The granuloma volume density was calculated as the quotient of the total granuloma profile area to the total sectional area studied per animal. The number of granulomas per unit volume of liver was assessed by applying the Weibel's (1969) formula. The sectional area of the red stained fibrous tissue was directly measured and calculated as a percentage of the total area examined, as previously described (Barbosa Jr., 2001; Coutinho et al., 2003).

\subsection{Biochemical study}

From each animal, a liver sample was frozen at $-70{ }^{\circ} \mathrm{C}$ for determination of collagen, measured as hydroxyproline by the Bergman and Loxley's (1963) spectrophotometric method B. Values for hepatic collagen were expressed in micromoles of hydroxyproline per gram of liver and corrected for intensity of infection by dividing the hydroxyproline content in the whole liver by the number of eggs per liver, results being expressed for 10,000s (Cheever, 1986).

\subsection{Parasitological study}

Worms recovered after perfusion of the portal system (Duvall and DeWitt, 1967) were counted and separated by sex. Quantification of the number of eggs in the liver tissue was performed after digestion in $4 \%$ potassium hydroxide (Cheever, 1968, 1970).

\subsection{Statistical analysis}

Data are presented as mean \pm S.E.M. Statistical significance was assessed by Student's $t$-test (when appropriate), one-way ANOVA with Tukey's and Tamhane's post hoc tests and the non-parametric Mann-Whitney's test (SPSS v.8 - Statistical Package for Social Sciences Inc., USA; and EXCEL - Microsoft, USA). Values of $p<0.05$ were taken to be significant.

\section{Results}

\subsection{Nutritional status}

Undernourished and well-nourished infected mice showed diverging growth curves, higher values having been detected for well-nourished animals. Undernourished mice did not show ascending values, as the controls (A2 and B2) but a slight weight gain could be noticed at the beginning of the experiment, followed by a tendency to a progressive loosing of weight until sacrifice. Effects of re-infection upon body weight gain were minimal and not dependent on the nutritional status of the mice, as can be seen in Fig. 1.

Measurements on food intake started during the first week of the trial over the total 22 weeks period, showing good correlation with body weight values.

\subsection{Morphological studies}

\subsubsection{Gross pathology}

The liver and spleen/body weight ratios from undernourished mice were always lower than those from 


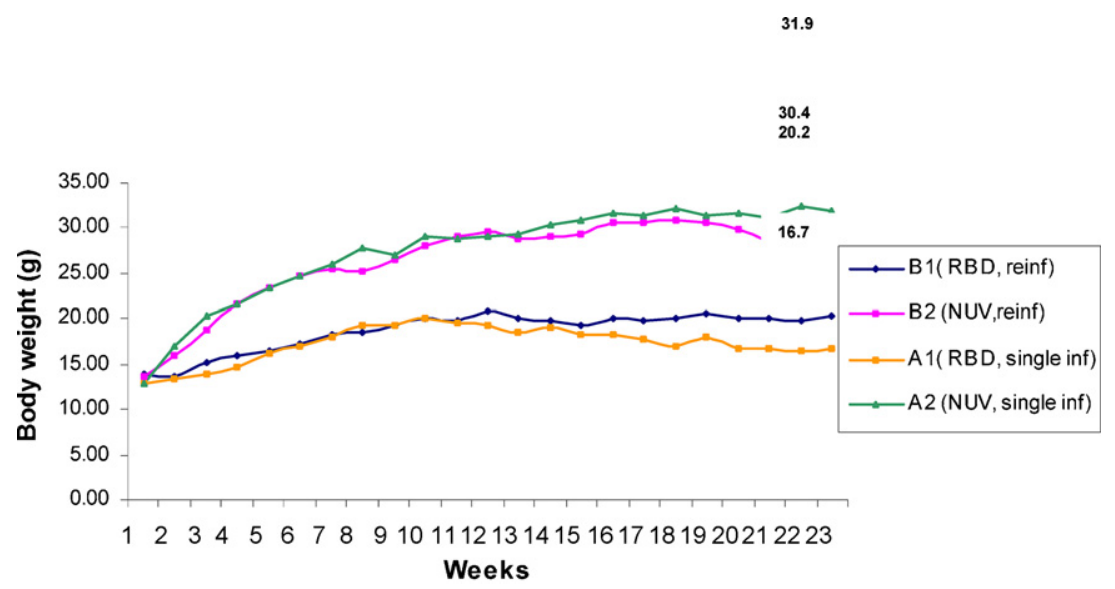

Fig. 1. Body weight of undernourished and well-nourished mice submitted to single and/or repeated infections with Schistosoma mansoni. Points on the curve represent the arithmetic mean of the group at each week of the trial. Numerical values on the growth curves refer to final mean body weight for each group. A1: undernourished mice, single infection; A2: well-nourished mice, single infection; B1: undernourished mice, re-infected five times; B2: well-nourished mice, re-infected five times.

their counterparts (well-nourished controls). Concerning liver weights, significant results were detected between undernourished and well-nourished mice, in both conditions of single infections or repeated infections, as well as between single infected and re-infected well-nourished animals. Undernourished mice, however, behave similarly either when single-infected or reinfected. Regarding spleen weights, a significant result was achieved between single-infected undernourished (A1) and well-nourished (A2) mice (Fig. 2).

\subsubsection{Histopathology}

Histologic examination showed only scattered small periovular granulomas (Fig. 3A) in undernourished single-infected mice (A1 group) and a mild non-specific reactional hepatitis (mononuclear and polymorphonu- clear eosinophils in some portal spaces and around central veins). Among well-nourished single infected mice (group A2), granulomas were larger (Fig. 3B), scattered distributed or grouped, delimited by thick collagen fibers (Fig. 3C). In this group only one animal developed the "pipestem"-like lesion, but in re-infected mice (group B2) 4 out of 16 animals showed portal concentration of periovular granulomas, associated with fibrotic expansion, development of thin fibrous tracts connecting portal spaces, and vascular neoformation, reproducing the histologic picture of murine "pipestem"-like fibrosis (Fig. 3D and E). In three additional animals a mixed histologic picture was detected (scattered circumoval granulomas and "pipestem"-like fibrosis). So, periportal fibrosis occurred in approximately $44 \%$ of the mice in group B2. Single infected and

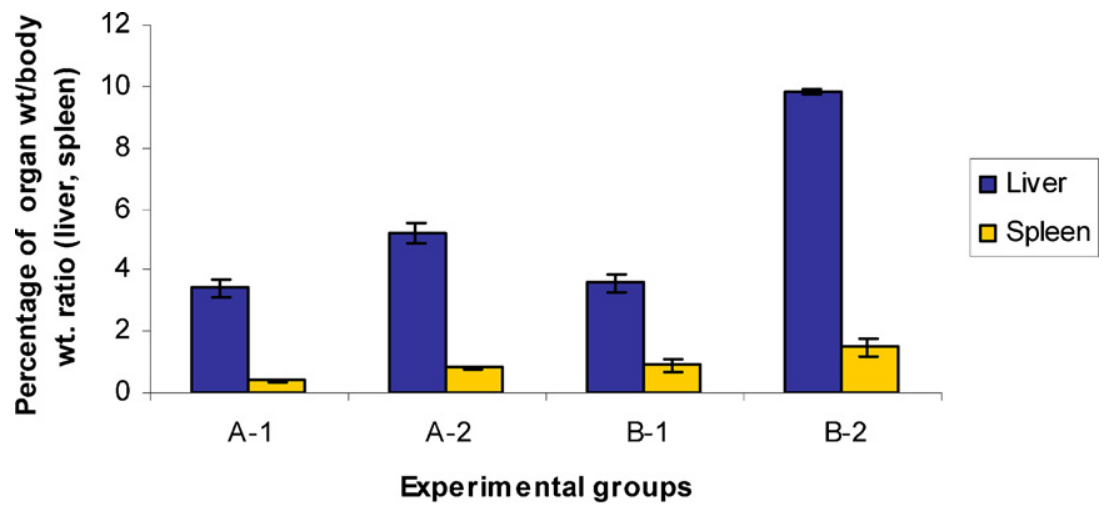

Fig. 2. Organomegaly (liver, spleen) in undernourished and well-nourished mice submitted to single and/or repeated infections with S. mansoni. A1: undernourished mice, single infection; A2: well-nourished mice, single infection; B1: undernourished mice, re-infected five times; B2: well-nourished mice, re-infected five times. 

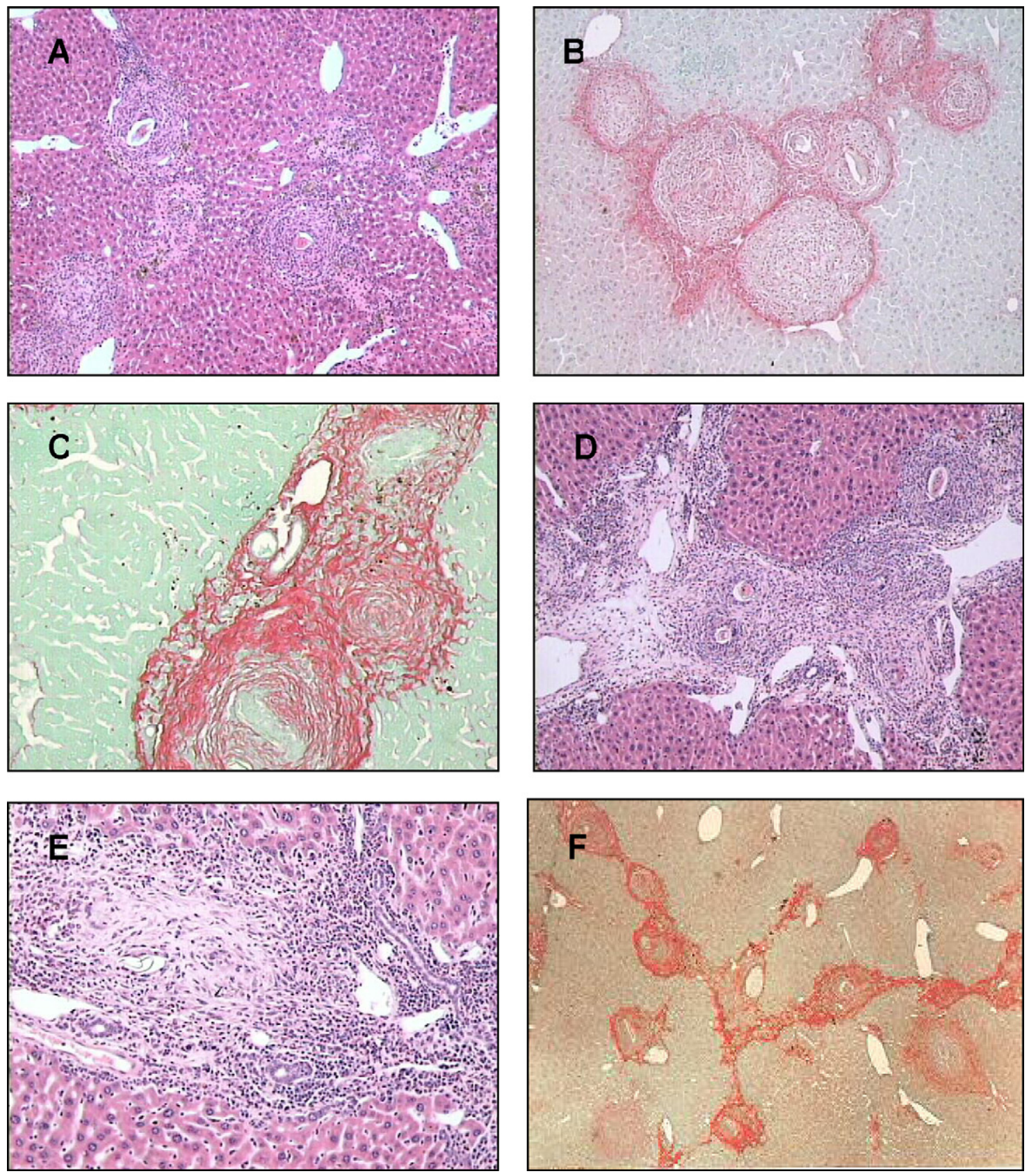

Fig. 3. Liver histopathology in single infected and re-infected mice: (A) scattered small periovular granulomas of the exudative type around degenerated eggs of S. mansoni (single infected undernourished mouse), hematoxylin and eosin 100×; (B) enlarged fibrotic granulomas around eggs of S. mansoni (single-infected well-nourished mouse), picrosirius-red 100×; (C) circumoval granulomas with deposition of dense collagen (single-infected well-nourished mouse), picrosirius-red 200×; (D) "pipestem" murine fibrosis in a re-infected well-nourished mouse (enlarged portal space with inflammatory infiltrate, periovular granulomas and vascular neoformation), hematoxylin and eosin 100×; (E) higher magnification of a fibrotic plaque in "pipestem"-like murine liver fibrosis (re-infected well-nourished mouse), hematoxylin and eosin 200×; (F) fibrous strands connecting scattered circumoval granulomas (re-infected undernourished mouse), picrosirius-red $100 \times$.

re-infected undernourished mice, however, did not show "pipestem"-like fibrosis, although thin fibrous strands could eventually be seen from one portal-space to the other (Fig. 3F).

\subsection{Morphometric analysis}

In each group (except in group A1 with 9 mice), ten random mice were submitted to morphometric analy- 


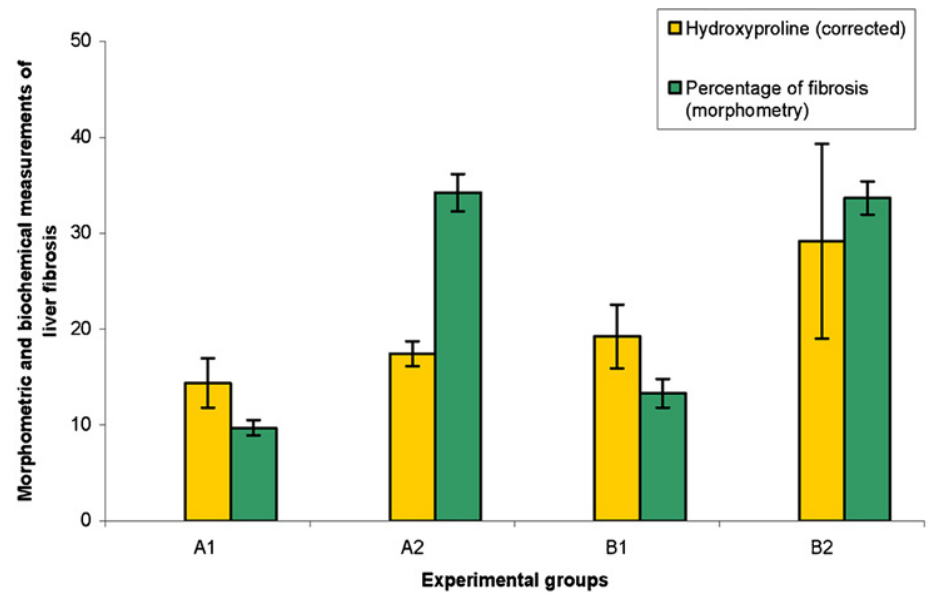

Fig. 4. Morphometric and biochemical evaluations of total liver fibrosis in undernourished and well-nourished mice with a single and/or multiple infections with $S$. mansoni. Values for hepatic collagen were initially determined as micromoles per gram of liver and then corrected for intensity of infection by dividing the hydroxyproline content in the whole liver by the number of eggs in the whole liver, results being expressed for 10,000 s (Cheever, 1986). A1: undernourished mice, single infection; A2: well-nourished mice, single infection; B1: undernourished mice, re-infected five times; B2: well-nourished mice, re-infected five times.

sis. Liver fibrosis measured as percentage of the hepatic tissue was markedly lower in single undernourished mice, with a slight but not significant increase seen in re-infected undernourished animals. A significant difference was noted between single-infected undernourished and well-nourished animals $(p=0.001)$. Differences between well-nourished single infected and re-infected mice, as well as between undernourished single infected and re-infected animals were not statistically significant (Fig. 4). Granuloma size was significantly higher
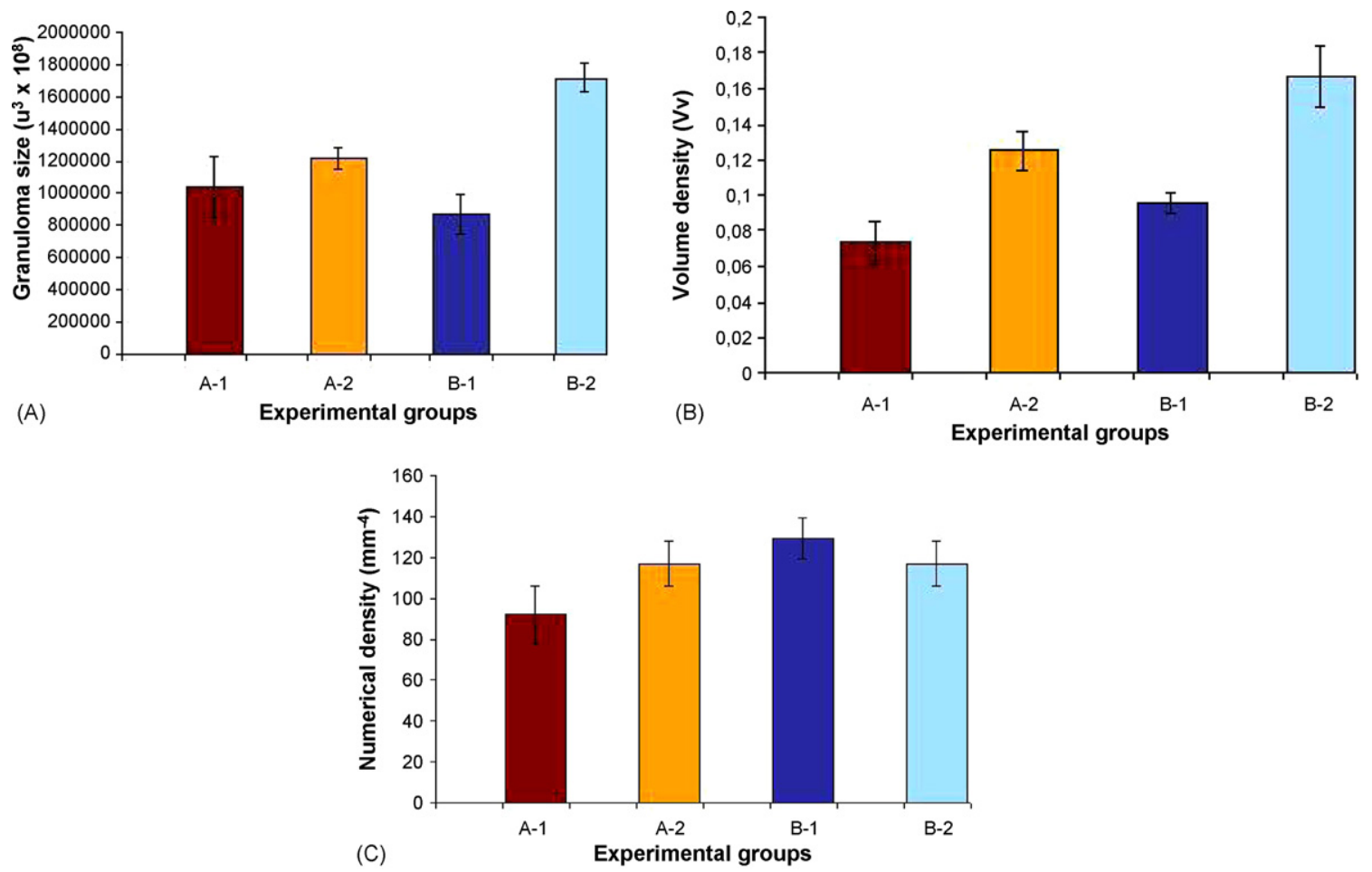

Fig. 5. Morphometric parameters (A, granuloma size; B, volume density; C, numerical density of circumoval granulomas) in the liver of undernourished and well-nourished mice submitted to single and/or repeated infections with S. mansoni. A1: undernourished mice, single infection; A2: well-nourished mice, single infection; B1: undernourished mice, re-infected five times; B2: well-nourished mice, re-infected five times. 
in re-infected well-nourished mice, when compared to single infected well-nourished and single or re-infected undernourished animals. Volume density $\left(V_{\mathrm{v}}\right)$ showed marked differences between undernourished and wellnourished mice, either single-infected or re-infected, with higher values for the re-infected well-nourished animals. Numerical density $\left(N_{\mathrm{n}}\right)$, however, did not differ significantly among the groups (Fig. 5A-C).

\subsection{Biochemical study}

Hydroxyproline levels were lower in undernourished mice, either single-infected or re-infected as compared to well-nourished controls. A significant difference was found between re-infected undernourished (B1) and well-nourished mice (B2) regarding collagen (hydroxyproline) content, with higher values for the last group. However, hydroxyproline content in the whole liver did not differ significantly between single-infected and reinfected groups. Regarding total liver fibrosis (Fig. 4), biochemical data showed good correlation with morphometric measurements.

\subsection{Parasitological data}

Total worm burdens appear in Fig. 6. Statistical differences were detected between single-infected and re-infected undernourished mice, as well as between single-infected undernourished and well-nourished animals.

Egg burdens calculated for the whole liver after tissue digestion showed highest values for re-infected mice (Fig. 6). Significant differences were also found between groups A1 (single-infected, undernourished) and A2 (single-infected, well-nourished), higher values for the latter; as well as between groups A1 (single-infected, undernourished) and B1 (re-infected, undernourished).

\section{Discussion}

Hepatic fibrosis, regardless of its cause, is a response to chronic liver injury, including that provoked by infection with $S$. mansoni. It is characterized by an increase in extracellular matrix constituents that collectively form the hepatic scar (Friedman, 1993). This scar consists of fibril forming collagens (Types I and III), as well as matrix glycoconjugates, including proteoglycans, fibronectin, and hyaluronic acid (Rojkind et al., 1979; Gressner and Bachem, 1990; Bissel, 1990). Current knowledge indicate that the fibrogenic cascade is initiated in the subendothelial space of Disse (Grimaud and Borojevic, 1977) and that the hepatic lipocyte (perisinusoidal or Ito cell) is the principal effector. In infected mice treated with Vitamin A, this cell was found in large numbers as a constituent of periovular granulomas and within areas of septal fibrosis (Barbosa Jr. et al., 1993).

In $S$. mansoni chronically infected mice, hepatic fibrosis is mainly associated with the circumoval granulomas (Dunn et al., 1977), although portal collagen deposition not associated with granulomas may be also present (Cheever, 1965). Normal mouse livers contain approximately $0.6 \mathrm{mg}$ of collagen per gm wet liver, whereas the $S$. mansoni-infected mouse livers contain about $10.3 \mathrm{mg} / \mathrm{g}$ wet liver, that represents a 17.6-fold increase (Wu et al., 1982). In the mouse model, repeated infections usually elicit experimental "pipestem"-like or periportal liver fibrosis in well-nourished animals and Santos et al. (2000), based on histological observations, reported the appearance of the lesion in $69.2 \%$ of re-infected mice versus $11.1 \%$ in single infected animals.



Fig. 6. Worm burden and liver tissue egg load, in undernourished and well-nourished mice submitted to single and repeated infections with $S$. mansoni. Values showed for egg columns must be multiplied by 1000. A1: undernourished mice, single infection; A2: well-nourished mice, single infection; B1: undernourished mice, re-infected five times; B2: well-nourished mice, re-infected five times. 
Since in previous investigations undernourished Swiss mice submitted to mild and prolonged infections and fed on a low-protein diet were unable to reproduce liver "pipestem" fibrosis (Coutinho et al., 1997), repeated infections were performed attempting to reproduce the lesion in this experimental host. Shifting from a deficient to a balanced diet and vice-versa were not able to induce the lesion in undernourished mice (Coutinho et al., 2003), as previously tried.

In the present experiment, undernourished mice did not develop "pipestem" fibrosis, although an increased number of well-nourished re-infected control mice (44\%) developed the lesion on histological examination. However, differences regarding collagen (hydroxyproline) concentration in the liver, detected by two different methods (morphometric and biochemical), were not significant when single infected and re-infected wellnourished mice were compared. The lack of association between occurrence of periportal fibrosis and higher levels of hydroxyproline in the liver of single infected BALB/c mice was reported by Silva et al. (2004).

The amount of collagen was always reduced in undernourished mice as compared to well-nourished animals, suggesting interference of the host nutritional status on the connective tissue changes of hepatic schistosomiasis in this animal model (Coutinho et al., 2003). It is known that dynamic deposition, accumulation and turnover of granuloma-associated matrix proteins occurs throughout experimental infection (Newport and Colley, 1993) and some still unclear factors and mechanisms may alter the total amount of liver fibrous tissue under different circumstances. Decreased total liver fibrosis in undernourished infected mice may result from an impaired mechanism of repair, a lower antigen load or a low production of pro-fibrotic cytokines, due to a low protein synthesis in a low-protein fed host (Oliveira et al., 2004).

In the present investigation the apparent lack of correlation between histological findings and morphometric/biochemical measurements of liver collagen in re-infected control mice is not surprising. In the chronic phase, most granulomas are immunologically modulated, reduced in size and their collagen content is not so abundant. Animals with overt periportal fibrosis usually display focal concentrations of collagen in enlarged portal spaces and less numerous scattered egg granulomas and so values detected by randomized morphometry could elicit misleading results. In effect, morphometric and biochemical measurements yielded similar results although not paralleling the histological findings regarding the occurrence of fibrosis.

Well-nourished and undernourished mice showed diverging growth curves, but the effects of re-infection upon host nutritional status in terms of body weight gain were minimal, variations being ascribed to the type of ingested diet.

Body, liver, and spleen weights seem to vary among different mouse strains and according to Fanning et al. (1981), more than one gene appears to be involved in the inheritance of the propensity to develop severe disease. In the present trial, organomegaly in undernourished mice was always lower than in well-nourished controls, when submitted either to single or repeated infections. Liver weights were most related to the nutritional status of the mice, but the number of infections (single, repeated) may influence more directly the degree of splenomegaly. Regarding granuloma volume and hepatic fibrosis, differences have been found among some inbred strains of well-nourished mice (Dunn et al., 1980), those strains with the largest granulomas usually showing most hepatic fibrosis. According to Cheever et al. (1987), the regulation of both granuloma size and hepatic fibrosis is complex, involving genes both outside and within the major histocompatibility complex, and is at least partly independent one another (Cheever, 1997). Previous experiment from our laboratories also showed the importance of the genetic background in the pathogenesis of periportal fibrosis since 4 out of 21 inbred undernourished C57BL/10 mice developed "pipestem" fibrosis when accidentally infected with a different parasite strain not usually employed in routine infection of mice in past experiments (Coutinho, 2004).

As expected, worm burdens were higher in wellnourished single infected mice. However, the amount of worms recovered from undernourished re-infected animals (group B1) did not differ significantly from that seen in well-nourished re-infected controls (group B2). So, it is likely that differences in liver pathology observed between the two groups were conditioned by the host response due to its nutritional status. Values concerning egg counts in hepatic tissue were presented for the whole liver, since difference in liver weight between undernourished mice and well-nourished controls could lead to misleading interpretation if data per gram of liver were used.

In this paper, attempts to induce periportal fibrosis in undernourished infected mice by repeated infections were not successful. Further investigations could focus, for instance, on the characteristics of the parasite strain to be used in mice infections, as well as on a deeper insight regarding immune response and immuneregulation in undernourished mice. A disregulation of fibrogenic cytokine production may account for why undernourished mice do not develop "pipestem" fibrosis. According to Wyler (1992), the failure to spontaneously 
down-regulate anti-schistosomal T-cell hypersensitivity and the resultant fibrogenic cytokine production may be an essential feature of those hosts who progress to severe liver fibrosis.

\section{References}

Andrade, Z.A., 1987. Pathogenesis of "pipestem" fibrosis of the liver (experimental observation on murine schistosomiasis). Mem. Inst. Oswaldo Cruz 82, 325-334.

Andrade, Z.A., Cheever, A.W., 1993. Characterization of the murine model of schistosomal hepatic periportal fibrosis ("pipestem" fibrosis). Int. J. Exp. Pathol. 74, 195-202.

Barbosa Jr., A.A., 2001. Morphological computer-assisted quantitative estimation of stained fibrous tissue in liver sections: applications in diagnosis and experimental research. J. Bras. Patol. 37, 197-200.

Barbosa Jr., A.A., Pfeifer, U., Andrade, Z.A., 1993. Role of fat-storing cells in schistosomal hepatic fibrosis of mice. Virchows Arch. B: Cell. Pathol. Incl. Mol. Pathol. 64, 91-96.

Bergman, I., Loxley, R., 1963. Two improved and simplified methods for the spectrophotometric determination of hydroxyproline. Anal. Biochem. 35, 1961-1965.

Bissel, D.M., 1990. Cell-matrix interaction and hepatic fibrosis. Prog. Liver Dis. 9, 143-155.

Cheever, A.W., 1965. A comparative study of Schistosoma mansoni infections in mice, gerbils, multimmate rats, and hamsters II Qualitative pathological differences. Am. J. Trop. Med. Hyg. 14, 229-238.

Cheever, A.W., 1968. Conditions affecting the accuracy of potassium hydroxide digestion technique for counting Schistosoma mansoni eggs in tissues. Bull. WHO 39, 328-331.

Cheever, A.W., 1970. Relative resistance of the eggs of human schistosomes to digestion in potassium hydroxide. Bull. WHO 43, 601-603.

Cheever, A.W., 1986. The intensity of experimental schistosoma infections modulates hepatic pathology. Am. J. Trop. Med. Hyg. 35, 124-133.

Cheever, A.W., 1997. Differential regulation of granuloma size and hepatic fibrosis in schistosome infections. Mem. Inst. Oswaldo Cruz 92, 689-692.

Cheever, A.W., Duvall, R.H., Hallack Jr., T.A., Minker, R.G., Malley, J.D., Malley, K.G., 1987. Variation of hepatic fibrosis and granuloma size among mouse strains infected with Schistosoma mansoni. Am. J. Trop. Med. Hyg. 37, 85-97.

Coura, J.R., 1975. Follow up of patients with schistosomiasis living in non-endemic area in Brazil. Bras. Med. 2, 45-47.

Coura, J.R., Wanke, B., Figueiredo, N., Argento, C.A., 1974. Evolutive pattern of schistosomiasis and life-span of Schistosoma mansoni in patients living in non-endemic area of Brazil. Rev. Soc. Bras. Med. Trop. 8, 193-198.

Coutinho, E.M., 1980. Patobiologia da desnutrição nas doenças parasitárias. Mem. Inst. Oswaldo Cruz 75, 63-76.

Coutinho, E.M., 2004. Malnutrition and hepatic fibrosis in murine schistosomiasis. Mem. Inst. Oswaldo Cruz 99 (Suppl. I), 85-92.

Coutinho, E.M., Barros, A.F., Barbosa Jr., A., Oliveira, S.A., Silva, L.M., Araújo, R.E., Andrade, Z.A., 2003. Host nutritional status as a contributory factor to the remodeling of schistosomal hepatic fibrosis. Mem. Inst. Oswaldo Cruz 98, 919-925.

Coutinho, E.M., Freitas, L.P.C.G., Abath, F.G.C., 1992. The influence of the regional basic diet from Northeast Brazil on health and nutri- tional conditions of mice infected with Schistosoma mansoni. Rev. Soc. Bras. Med. Trop. 25, 13-20.

Coutinho, E.M., Souza, M.M., Silva, L.M., Cavalcanti, C.L., Araújo, R.E., Barbosa Jr., A.A., Cheever, A.W., Andrade, Z.A., 1997. Pathogenesis of schistosomal "pipestem" fibrosis: a low-protein diet inhibits the development of "pipestem" fibrosis in mice. Int. J. Exp. Pathol. 78, 337-341.

Dunn, M.A., Cheever, A.W., Dean, D.A., Duvall, R.H., Kelley, E.P., 1980. Diminished liver fibrosis in murine schistosomiasis in an inbred mouse strain. Clin. Res. 28, 274A (Abstract).

Dunn, M.A., Rojkind, M., Warren, K.S., Hait, P.K., Rifas, L., Seifter, S., 1977. Liver collagen synthesis in murine schistosomiasis. J. Clin. Invest. 59, 666-674.

Duvall, R.H., DeWitt, W.B., 1967. An improved perfusion technique for recovering adult schistosomes from laboratory animals. Am. J. Trop. Med. Hyg. 16, 483-486.

Fanning, M.M., Peters, P.A., Davis, R.S., Kazura, J.W., Mahmoud, A.A.F., 1981. Immunopathology of murine infection with Schistosoma mansoni: relationship of genetic background to hepatosplenic disease and modulation. J. Infect. Dis. 144, 148-153.

Friedman, S.L., 1993. The cellular basis of hepatic fibrosis. Mechanisms and treatment strategies. N. Engl. J. Med. 328, 18281835 .

Gressner, A.M., Bachem, M.G., 1990. Cellular sources of noncollagenous matrix proteins: role of fat-storing cells in fibrogenesis. Semin. Liver Dis. 10, 30-46.

Grimaud, J.A., Borojevic, R., 1977. Chronic human Schistosomiasis mansoni: pathology of the Disse's space Lab. Investigation 36, 268-273.

Henderson, G.S., Nix, N.A., Montesano, M.A., Gold, D., Freeman Jr., G.L., McCurley, T.L., Colley, D.G., 1993. Two distinct pathological syndromes in male CBA/J inbred mice with chronic Schistosoma mansoni infections. Am. J. Pathol. 142, 703-713.

Junqueira, L.C.U., Bignolas, G., Brentani, R., 1979. Picrosirius staining plus polarization microscopy, a specific method for collagen detection in tissue section. Histochem. J. 11, 447-455.

Newport, G.R., Colley, D.G., 1993. Schistosomiasis. In: Warren, K.S. (Ed.), Immunology and Molecular Biology of Parasitic Infections, 3rd ed. Blackwell Science Publication, Boston, pp. 387-437.

Oliveira, S.A., Silva, L.M., Barbosa Jr., A.A., Ribeiro-dos-Santos, R., Coutinho, E.M., Andrade, Z.A., Soares, M.B.P., 2004. Decreased humoral and pathologic responses in undernourished mice infected with Schistosoma mansoni. Parasitol. Res. 93, 30-35.

Potter, J.J., Mezey, E., 2002. Leptin deficiency reduces but does not eliminate the development of hepatic fibrosis in mice infected with Schistosoma mansoni. Liver 22, 173-177.

Potter, J.J., Rennie-Tankesley, L., Mezey, E., 2003. Influence of letptin in the development of hepatic fibrosis produced in mice by Schistosoma mansoni infection and by chronic carbon tetrachloride administration. J. Hepatol. 38, 281-288.

Rojkind, M., Giambrone, M.-A., Biempica, L., 1979. Collagen types in normal and cirrhotic liver. Gastroenterology 76, 710-719.

Santos, A.B.A., Souza, M.M., Andrade, Z.A., 2000. Reinfecções e desenvolvimento da fibrose periportal esquistossomótica no modelo murino. Rev. Soc. Bras. Med. Trop. 33, 197-200.

Silva, L.M., Oliveira, S.A., Ribeiro-dos-Santos, R., Andrade, Z.A., Soares, M.B.P., 2004. Comparison of immune response of Schistosoma mansoni-infected mice with distinct chronic forms of the disease. Acta Trop. 9, 189-196.

Symmers, W.St.C., 1904. Note on a new form of liver cirrhosis due to the presence of the ova of Bilharzia haematobia. J. Pathol. Bacteriol. 9, 237-239. 
Teodósio, N.R., Lago, E.S., Romani, S.A.M., Guedes, R.C.A., 1990. A regional basic diet from Northeast Brazil as a dietary model of experimental malnutrition. Arch. Lat. Am. Nutr. 40, 533-547.

von Lichtemberg, L., Sadun, E.H., 1968. Experimental production of bilharzial "pipestem" fibrosis in the chimpanzee. Exp. Parasitol. 22, 264-278.

Warren, K.S., 1966. The pathogenesis of clay pipestem cirrhosis in mice with chronic Schistosomiasis mansoni, with a note on the longevity of the schistosomes. Am. J. Pathol. 49, 479-489.
Weibel, E.R., 1969. Stereological principles for morphometry in electron microscopic cytology. Int. Rev. Cytol. 26, 235302.

Wu, C.H., Giambrone, M.-A., Howard, D.J., Rojkind, M., Wu, G.Y., 1982. The nature of the collagen in hepatic fibrosis in advanced murine Schistosomiasis. Hepatology 2, 366-371.

Wyler, D.J., 1992. Molecular and cellular basis of hepatic fibrogenesis in experimental Schistosomiasis mansoni infection. Mem. Inst. Oswaldo Cruz 87 (Suppl. IV), 117-125. 